\title{
Lusioersily
}

\section{A bacterial foraging optimization and learning automata based feature selection for motor imagery EEG classification}

Pal, M., Bhattacharyya, S., Roy, S., Konar, A., Tibarewala, D. N., \& Janarthanan, R. (2014). A bacterial foraging optimization and learning automata based feature selection for motor imagery EEG classification. In 2014 International Conference on Signal Processing and Communications, SPCOM 2014 [6983926] (2014 International Conference on Signal Processing and Communications, SPCOM 2014). Institute of Electrical and Electronics Engineers Inc.. https://doi.org/10.1109/SPCOM.2014.6983926

Link to publication record in Ulster University Research Portal

Published in:

2014 International Conference on Signal Processing and Communications, SPCOM 2014

Publication Status:

Published (in print/issue): 12/12/2014

DOI:

10.1109/SPCOM.2014.6983926

Document Version

Author Accepted version

\section{General rights}

Copyright for the publications made accessible via Ulster University's Research Portal is retained by the author(s) and / or other copyright owners and it is a condition of accessing these publications that users recognise and abide by the legal requirements associated with these rights.

\section{Take down policy}

The Research Portal is Ulster University's institutional repository that provides access to Ulster's research outputs. Every effort has been made to ensure that content in the Research Portal does not infringe any person's rights, or applicable UK laws. If you discover content in the Research Portal that you believe breaches copyright or violates any law, please contact pure-support@ulster.ac.uk. 


\title{
A Bacterial Foraging Optimization and Learning Automata Based Feature Selection for Motor Imagery EEG Classification
}

\author{
Monalisa Pal $^{1, \text { a }}$, Saugat Bhattacharyya ${ }^{1, \mathrm{~b}}$, Shounak Roy ${ }^{2, \mathrm{c}}$, Amit Konar ${ }^{1, \mathrm{~d}}$, D.N. Tibarewala ${ }^{3, \mathrm{e}}$, R. \\ Janarthanan ${ }^{4, f}$ \\ ${ }^{1}$ Dept. of Electronics \&Telecommunication Engg. ${ }^{2}$ Electronic Systems Engineering, ${ }^{3}$ School of Bioscience \& \\ Engg., ${ }^{4}$ Dept. of Computer Science. \\ ${ }^{1,3}$ Jadavpur University, ${ }^{2}$ Indian Institute of Science, ${ }^{4} \mathrm{TJS}$ Engineering College. \\ ${ }^{1,3}$ Kolkata, ${ }^{2}$ Bangalore, ${ }^{4}$ Chennai, India. \\ amonalisap90@gmail.com, bsaugatbhattacharyya@live.com, 'shounakroy2009@gmail.com, \\ dkonaramit@yahoo.co.in, ${ }^{\mathrm{d}}$ biomed.ju@gmail.com, ${ }^{\mathrm{f}}$ srmjana_73@yahoo.com.
}

\begin{abstract}
Selection of relevant features is an open problem in Brain-computer interfacing (BCI) research. Sometimes, features extracted from brain signals are high dimensional which in turn affects the accuracy of the classifier. Selection of the most relevant features improves the performance of the classifier and reduces the computational cost of the system. In this study, we have used a combination of Bacterial Foraging Optimization and Learning Automata to determine the best subset of features from a given motor imagery electroencephalography (EEG) based BCI dataset. Here, we have employed Discrete Wavelet Transform to obtain a high dimensional feature set and classified it by Distance Likelihood Ratio Test. Our proposed feature selector produced an accuracy of $80.291 \%$ in 216 seconds.
\end{abstract}

Index Terms - Discrete Wavelet Transform, Brain-Computer Interfacing, Bacterial Foraging Optimization Algorithm, Learning Automata, Distance Likelihood Ratio Test.

\section{INTRODUCTION}

$\mathrm{B}$ rain-Computer Interfacing (BCI) has been a widely investigated technology in neuro-rehabilitative research. It aims to provide disabled individuals a non-muscular pathway between brain (generates motor intentions) and a prosthetic device (implementing motor intentions). Such applications are not only limited to rehabilitation but also extends to virtual gaming, tele-operation, communication, robotics, etc. [1-2]. The basic steps of BCI include acquisition of brain signals, pre-processing, feature extraction and classification of extracted features whose success is followed by developing a control strategy for application to an external device [3-4].

Electroencephalography (EEG) is the most widely used brain signal measuring device because of its non-invasiveness, easy availability, portability and high temporal resolution [5]. After signal acquisition, the signal is pre-processed and relevant features are extracted. Often the dimension of the feature space is very large having redundant features which not only creates additional overhead of managing the space complexity but also might include outliers, thereby reducing classification accuracy [6].

Literature galore with studies on EEG-based BCI that have successfully discriminated between left-right motor imagery signals [7-8]. Recently, the trend has shifted to wrist and finger movement classification from EEG [9-10]. In [11], researchers claim that the EEG modality during movement imagination or execution is Event Related Desynchronization and Synchronization (ERD/S). Recently, Principal Component Analysis (PCA) [12], Independent Component Analysis (ICA) [13] and Sequential Forward Search (SFS) [14] has been used for feature selection. Here, the number of features selected are not user-defined but is based on the best subset of features determined by the algorithm. Evolutionary Algorithms can deal with this by selecting the best subset of features. Differential Evolution (DE) [15], Firefly Algorithm (FA) [16], Particle Swarm Optimization (PSO) [17] and Artificial Bee Colony (ABC) Optimization [18] etc. have also found application in BCI.

In this study, we have used EEG-based BCI to model the intention of grasping activity for which we have discriminated between extension and flexion of wrist and opening and closing of fist. Here, Discrete Wavelet Transform is used to decompose the EEG data and detail coefficients at level 3 (D3) and 4 (D4) are used as features. This creates a very large feature space on which feature selection is applied, followed by classification using Distance Likelihood Ratio Test (DLRT). For feature selection, classical Bacterial Foraging Optimization (BFO) has been used because of its capability in finding approximate solutions of extremely difficult optimization problems like the Rastrigin function problem. However, using BFO algorithm alone results in local optima which is avoided by the use of Learning Automata. This also allows us to vary the step size of BFO at quantized levels which is the novelty of our work. 
The organization of the rest of the paper is as follows. Section II describes the experiments and the philosophy behind the entire work. The results are discussed in Section III. Finally, Section IV concludes the paper giving a brief summary of the work and mentioning future scope of the research.

\section{EXPERIMENTS AND METHODS}

This section gives a brief description of the processing of the raw EEG and the classifier applied in this study, highlighting our proposed feature selection scheme.

\section{A. Stimuli Presentation}

The EEG signal is collected from 8 healthy, right-handed subjects, 4 male and 4 female in the age group of $25 \pm 3$ years, in 3 sessions. The data acquisition consists of instructing the subjects through a sequence of visual stimulus or commands asking the subject to imagine the execution of the corresponding motor imagery task, which is, extension/flexion of the wrist and opening and closing of the fingers.

Fig. 1 shows the generic structure of the visual cue. During the initial blank screen, the subject relaxes which provides the baseline of the EEG. The ready command instructs the subject to get ready to imagines performing a task. Next, the subject imagines the task based on the command displayed on screen for 3 seconds. For the opening and closing commands, the subject imagines opening and closing his/her fist. During flexion and extension commands, the subject imagines bending his/her palm towards the arm and moving the palm away from the arm, respectively, thereby modelling the action required for displacing the object. The complete process is repeated 50 times for each of the four kinaesthetic tasks in random fashion to obtain non-overlapping and unbiased EEG responses.

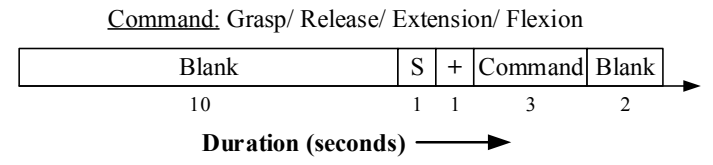

Fig. 1. Stimulus for EEG acquisition

\section{B. Data Acquisition and Pre-processing}

Informative motor imagery signals predominantly appear in the primary, supplementary and pre-motor cortex region of the brain and is recorded from $\mu(8-12 \mathrm{~Hz})$ and central $\beta(16-24$ $\mathrm{Hz}$ ) band of EEG signals [11]. EEG is acquired with the help of a 14 channel Emotiv headset having a sampling rate of 128Hz. The electrodes: AF3, F7, F3, FC5, T7, P7, O1, O2, P8, T8, FC6, F4, F8 and AF4, are set up according to the standard 10/20 system of electrode placement method [19].

To obtain the required information the EEG signal of 8-25 $\mathrm{Hz}$ bandwidth is filtered to remove other form of environmental and other cognitive noises from the signal. We have used a 12th order elliptical filter of $1 \mathrm{~dB}$ passband ripple and $50 \mathrm{~dB}$ stopband ripple for this purpose. The merit of elliptical filter is its sharp roll-off characteristics and equiripple behavior in the passband and the stopband as compared to the other standard filters [20].
To remove the effect of cross-talk from neighbouring electrodes, a spatial filtering method is required. Common average referencing [19] is done on the raw data. It subtracts the mean voltage of the 14 electrodes from the signal of each of the electrodes.

\section{Feature Extraction: Discrete Wavelet Transform (DWT)}

Wavelet transform provides both frequency as well as timedomain analysis and at multiple resolution, unlike Fourier Transform which provides frequency domain analysis at a constant resolution on the frequency scale. In DWT, the signals are passed through filters with different cutoff frequencies and different scales. The number of filter stages (levels) to be used depends on the resolution required.

We have prepared the feature vector using the detail coefficients of third and fourth level (D3 and D4) for each electrode because these levels contains information in the frequency range of $8-12 \mathrm{~Hz}$ and $16-24 \mathrm{~Hz}$. In this study, we have selected Daubechies wavelet of order $4(\mathrm{db} 4)$ as the mother wavelet to decompose the incoming EEG data. Considering 14 electrodes, the dimension of a feature-vector is 1176. The features are normalized in the range $[0,10]$. Further details on wavelet transforms can be found on [21].

\section{Our Proposed Feature Selection Scheme: BFO-LA}

This work uses Bacterial Foraging Algorithm [22] whose step size C is selected by the use of Learning Automata [23]. A brief description of the scheme is presented, here.

\section{1) Initialization:}

For selecting a subset from $D$-dimensional features, the population members are randomly initialised as a $2 D$ dimensional vector $\theta_{j}=\left\{w_{l j}, \ldots, w_{2 D j} ; w_{i j} \in[0,1]\right\}$ for $j=1,2$, $\ldots, N P$, where the first $D$ components i.e. for $i=1,2, \ldots, D$, represents the Activation Thresholds and the last $D$ components i.e. for $i=D+1, D+2, \ldots, 2 D$ represents the Scaling Factors. The selection of the subset of feature at any iteration is governed by (1) and shown in Fig. 2.

$$
\begin{aligned}
& \text { If }\left(w_{i j}>0.5\right) \\
& \text { feat }_{i} \leftarrow w_{(i+D) j} \times f_{i} \quad, \text { feat }_{i} \in S_{m} \\
& \text { else } \\
& \text { ignore feat } \\
& \text { ign }
\end{aligned}
$$

where $i=1,2, \ldots, D$. For our work, $D$ is equal to 1176 . This indicates that if Activation of the $i^{\text {th }}$ component of the $j^{\text {th }}$ member at state $m$ is higher than 0.5 , then the corresponding feature is scaled by the Scaling Factor (Linear Scaling increases discrimination power of classifier). Scaling Factors remain constant over train-set, validation-set and test-set at every state to avoid misinterpretation of the data.

The state transition probability matrix is initialized uniformly with 0.05 (due to lack of a priori information all values are equally likely) for the parameter $C$ of BFO at 20 quantized levels $\left(C_{1}, C_{2}, \ldots, C_{20}\right)$ between $(0,1]$. Thus, $S_{m}$ is of the order $N P \times 20$. 


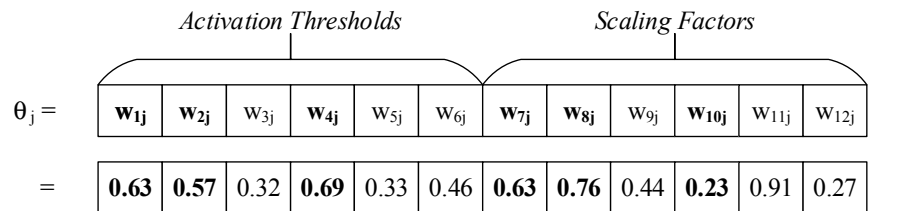

feat $_{j}(m)=\left\{0.63 \times f_{1}, 0.57 \times f_{2}, 0.69 \times f_{4}\right\}$

Fig. 2. Vector representation of the $j^{\text {th }}$ population member with 6 features at state $S_{m}$. Activation Thresholds $>0.5$ and the corresponding Scaling Factors are shown in bold. The selected subset is also mentioned as feat ${ }_{j}$.

\section{2) Adaptive Selection of Parameters for $B F O$}

For selecting $C_{j}$ for the $i^{\text {th }}$ population member, a random number $r$ between $(0,1)$ is generated and the selection of $C_{j}$ is done using (2).

$$
\sum_{m=1}^{j-1} p\left(C_{m}\right)<r \leq \sum_{m=1}^{j} p\left(C_{m}\right)
$$

\section{3) Bacterial Foraging Algorithm (BFO)}

The principal steps of foraging by E.coli bacteria are: chemotaxis, swarming, reproduction and eliminationdispersal.

a) Chemotaxis: During chemotaxis in a nutrient medium, an E.coli bacterium $\theta_{i}$ tumbles an unit step, $C(i)$ in a random direction, given by a random vector $\Delta(i) \in R^{2 D}$ whose each component is between $[-1,1]$. This tumbling behavior is given by (3). If this is found to be favorable then $\theta_{i}$ swims for a period of time (swim length, $N_{s}$ ) in that direction. The number of chemotactic steps is determined by $N_{c}$.

$$
\theta_{i}(j+1, k, l)=\theta_{i}(j, k, l)+C(i) \frac{\Delta(i)}{\sqrt{\Delta^{T}(i) \Delta(i)}}
$$

b) Swarming: When an E.coli cell moves up the nutrient gradient, it releases an attractant. Due to this, several cells of E.coli form stable spatio-temporal patterns of concentric rings (swarms). The cell-to-cell signaling $J_{c c}$ by a bacteria $\theta$ to the total population $P$ is given by (4). This is added to the fitness function $J(\theta)$ to provide a time-varying fitness.

$$
\begin{aligned}
& J_{C C}(\theta, P(j, k, l))=\sum_{i=1}^{N P} J_{C C}\left(\theta, \theta_{i}(j, k, l)\right) \\
& =\sum_{i=1}^{N P}\left[-d_{\text {atrractant }} \exp \left(-w_{\text {attractant }} \sum_{m=1}^{2 D}\left(\theta^{m}-\theta_{i}^{m}\right)^{2}\right)\right] \\
& +\sum_{i=1}^{N P}\left[h_{\text {repellant }} \exp \left(-w_{\text {repellant }} \sum_{m=1}^{2 D}\left(\theta^{m}-\theta_{i}^{m}\right)^{2}\right)\right]
\end{aligned}
$$

where $d_{\text {attractant }}, w_{\text {attractant }}, h_{\text {repellant }}$ and $w_{\text {repellant }}$ are the different coefficients.

c) Reproduction: From the total population, the least healthy half dies (deleted) and the healthiest half asexually splits into two (copied) to keep the population size constant. It is to be noted, here, that higher value of $J(\theta)$ means lower health of the bacteria $\theta$. This iterates for $N_{r e}$ steps. After every reproduction phase, chemotaxis and swarming repeats.

d) Elimination-dispersal: Sometimes, sudden change in environment kills a few bacteria and to balance this nature disperses some bacteria at a new location. For simulation, a bacteria is eliminated with probability $p_{e d}$. If a bacterium is eliminated, another bacteria is dispersed randomly at any location on the optimization domain. This continues for $N_{e d}$ steps where every step is followed by chemotaxis, swarming and reproduction.

\section{4) Updating State Transition Probability Matrix}

At state $S_{m}$, on selecting a subset of features using the $k^{\text {th }}$ member $\theta_{k}$ having fitness $J_{k}$ which was updated in BFO using $C_{j}$, the state transition probabilities are updated using Linear Reinforcement Scheme [23] given by (5) and (6).

If $J_{k}\left(\theta_{k}\left(C_{j}, m\right)\right)<J_{k}\left(\theta_{k}(m-1)\right)$

$$
\left\{\begin{array}{c}
p_{i}(m+1)=(1-a) p_{i}(m) \quad \forall i \neq j \\
p_{j}(m+1)=p_{j}(m)+a\left(1-p_{j}(m)\right)
\end{array} \quad \forall\right.
$$

Otherwise,

$$
\left\{\begin{array}{c}
p_{i}(m+1)=\frac{b}{e-1}+(1-b) p_{i}(m) \quad \forall i \neq j \\
p_{j}(m+1)=(1-b) p_{j}(m)
\end{array}\right.
$$

where $a \in[0,1]$ is the reward response, $b \in[0,1]$ is the penalty response and $e$ is the number of actions of the automata process. Generally, $a$ and $b$ are equal. In this study, we have used $a=b=0.1$.

\section{5) Fitness Evaluation and State Assignment}

The fitness $\left(J_{j}\right)$ of the $j^{\text {th }}$ population member is evaluated on the basis of classification accuracy $\left(C A_{v}\right)$ evaluated using Validation-set with DLRT trained on the Train-set. The fitness function is given by (7) as thus, we have a minimization problem.

$$
J_{j}=1 / C A_{v}
$$

Following state assignment and fitness evaluation, the population members are sorted in the ascending order of fitness and their corresponding states are assigned to them.

6) Pseudo-code for Feature Selection

The pseudo-code for feature selection is given here.

- Step 1: A set of $N P$ vectors each with $2 D$ components is randomly initialized between 0 and 1 .

- Step 2: Features are selected based on fitness according to rule (7) for every population member (1 to $N P$ ).

- Step 3: DLRT is trained on Train-set with selected feature set.

- Step 4: The result and the choice of parameters are validated using the Validation-set and fitness of population members is re-evaluated.

- Step 5: Population members are updated according to the BFO algorithm guided by the fitness values calculated in the previous step. 
- Step 6: if gen gen $_{\max }$ goto Step 2, else select member with the best fitness (first population member) to get the final set of features.

\section{E. Classification: Distance Likelihood Ratio Test}

We aim for a two-level classification, where at each level a binary classification is performed as shown in Fig. 3. The classifier solves dual purpose of assisting in the evaluation of the fitness function during feature selection as well as in the assessment of the performance of our approach.

Likelihood Ratio, $R$, as derived from Bayes' theorem is given by (8). If $R$ is greater than 1, an unknown sample $x$, is assigned to class $C_{0}$, otherwise it is assigned to class $C_{l}$. Distance Likelihood Ratio Test (DLRT) uses non-parametric estimation for the distribution of the features for a given class, using which $R$ simplifies to (9). This ratio serves as the threshold or the decision boundary for classification [14, 24].

$$
\begin{gathered}
R=\frac{P\left(C_{0} \mid x\right)}{P\left(C_{1} \mid x\right)}=\frac{P\left(C_{0}\right) p\left(x \mid C_{0}\right)}{P\left(C_{1}\right) p\left(x \mid C_{1}\right)} \\
R=\frac{n_{C_{1}}}{n_{C_{0}}}\left(\frac{\Delta_{k^{(1)}}}{\Delta_{k^{(0)}}}\right)^{d}
\end{gathered}
$$

where $\Delta_{k}^{(i)}$ is the distance of the $k$-th neighbor in class $C_{i}$ $(i=0,1), d$ is the dimensionality of the feature space and ${ }^{n} C_{i}$ is the fraction of sample of class $C_{i}$ within the considered neighborhood.

Fig. 3 outlines the major steps of the proposed work. The raw EEG data is filtered and detail wavelet coefficients at level 3 and 4 are used to construct the feature space. The feature set is 10-fold cross-validated, and Train-set, Validation-set and Test-set are prepared with $70 \%, 15 \%$ and $15 \%$ samples respectively. The level-1 classifiers indicate which body part the movement is related to and the level-2 classifiers classify the movements of the body part determined at level-1.

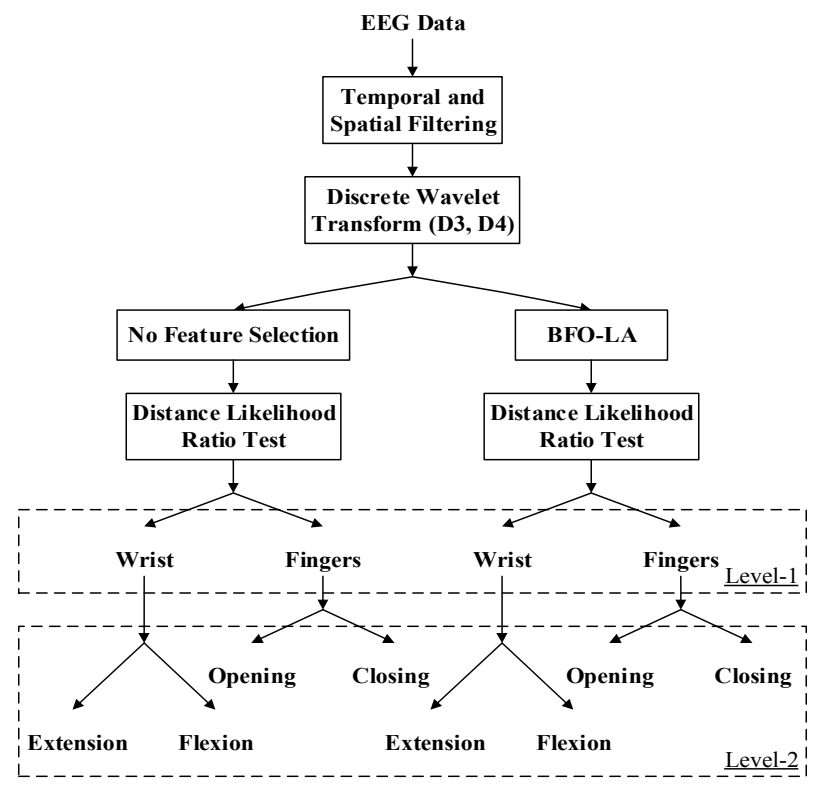

Fig. 3. Flowchart of the proposed scheme

\section{RESULTS AND DISCUSSION}

The proposed work is carried out on MATLAB R2012b environment using an Intel Core i3 CPU @ 2.30 GHz having 3.82GB RAM and 64-bit Windows 8 Operating System.

The average classification accuracies (CA) and the computation time (CT, from pre-processing to classification) measured over 10 runs and over 3 sessions for each of the eight subjects considering all the classifiers at both the levels are as shown in Table I which shows significant improvement in the performance.

TABLE I

Performance OVER 10 Runs (S.D. - Standard DeVIATION)

\begin{tabular}{llllll}
\hline \hline \multirow{2}{*}{ Subject ID } & \multicolumn{2}{l}{ NO FEATURE SELECTION } & & \multicolumn{2}{c}{ BFO-LA } \\
\cline { 2 - 3 } \cline { 5 - 6 } & \multicolumn{1}{c}{ CA $(\%)$} & CT $(\mathrm{s})$ & & CA $(\%)$ & CT $(\mathrm{s})$ \\
\hline 1 & 77.085 & 1.6549 & & 82.915 & 212.65 \\
2 & 76.665 & 1.6083 & & 80.415 & 224.27 \\
3 & 73.75 & 1.6031 & & 78.585 & 236.43 \\
4 & 77.085 & 1.6437 & & 82.500 & 192.48 \\
5 & 71.25 & 1.6341 & & 77.085 & 196.52 \\
6 & 75.835 & 1.5988 & & 82.415 & 219.53 \\
7 & 72.915 & 1.6382 & & 77.165 & 203.83 \\
8 & 78.335 & 1.6183 & & 81.250 & 239.93 \\
\hline Mean & 75.365 & 1.625 & 80.291 & 215.705 \\
S. D. & 2.455 & 0.021 & 2.395 & 17.575 \\
\hline \hline
\end{tabular}

The performance of BFO-LA is compared with other competitor algorithms: DE [15], PSO [17], ABC [18] and FA [16]. The parameters of selected for these algorithms are mentioned in Table II. The average value of $C A, C T$ and the number of features selected (FS) from 1176 features for different algorithms are noted in Table III from which we note that BFO-LA provides best results in terms of $C A$ and $F S$. Although, $C T$ of FA is minimum, the time taken by BFO-LA is not very high. Thus, BFO-LA provides optimal results trading of with time and accuracy.

TABLE II

PARAMETERS USED IN COMPETITOR ALGORITHMS

\begin{tabular}{ll}
\hline \hline Algorithm & Parameters \\
\hline DE & $N P=50$, Cross-over Probability $=0.9$, Scale Factor $F=0.9$ \\
PSO & $N P=50$, Inertia Factor $\omega=0.7$, Acceleration Coefficients \\
& $C_{l}=C_{2}=2$ \\
ABC & $N P=50$, Limit Cycle $=50$ \\
FA & $N P=50$, Maximum Attractiveness $\beta_{0}=1$, Light Absorption \\
& Coefficient $\gamma=2$ \\
BFO-LA & $N P=50, d_{\text {attract }}=h_{\text {repellant }}=0.1, w_{\text {attract }}=0.2, w_{\text {repellant }}=10, N_{c}=20$, \\
& $N_{s}=5, N_{r e}=2, N_{e d}=2, p_{e d}=0.025$ \\
\hline \hline
\end{tabular}

TABLE III

\begin{tabular}{|c|c|c|c|}
\hline \multirow{2}{*}{ Algorithms } & \multicolumn{3}{|c|}{ Performance Metrics } \\
\hline & CA $(\%)$ & $\mathrm{CT}(\mathrm{s})$ & FS \\
\hline $\mathrm{DE}$ & 72.277 & 197 & 603 \\
\hline PSO & 71.050 & 225 & 621 \\
\hline $\mathrm{ABC}$ & 74.334 & 221 & 618 \\
\hline FA & 78.423 & 179 & 590 \\
\hline BFO-LA & 80.291 & 216 & 585 \\
\hline
\end{tabular}

COMPARISON WITH COMPETITOR ALGORITHMS

\section{CONClusion AND Future Direction}

This study proposes a feature selection algorithm using Bacterial Foraging Optimization (BFO) Algorithm for its 
merit of complex multi-optimal function optimization and implements Learning Automata (LA) to overcome the problem of entrapping in local minima. The proposed algorithm selects a subset of 1176 features extracted from EEG data set using discrete wavelet transform. EEG has been acquired from 8 subjects for 3 sessions for four motor imagery tasks which model the grasping related motor intentions: opening/closing of fist and extension/flexion of wrist. The selected features are classified using Distance Likelihood Ratio Test. BFO-LA feature selection reduces the feature set to 585 dimension providing an accuracy of $80.291 \%$ in 216 seconds. This is a good trade-off between accuracy and computational time thereby delivering optimal results.

In our future work, we would like to classify other finer movements of wrist and fingers. Our future goal is to apply this method in controlling a robot arm in a real-time scenario. This can further assist as a rehabilitative tool to increase the functionality of a disabled person.

\section{ACKNOWLEDGMENT}

This study has been supported by University Grants Commission (UGC), India; University with Potential for Excellence Program (UGC-UPE) (Phase II) in Cognitive Science, Jadavpur University and Council of Scientific and Industrial Research (CSIR), India.

\section{REFERENCES}

[1] D. J. McFarland, J. R. Wolpaw, "Brain-Computer Interface Operation of Robotic and Prosthetic Devices", IEEE Computer Society, 2008.

[2] J. J. Daly, J. R. Wolpaw, "Brain-computer interfaces in neurological rehabilitation", Lancet Neurol, vol. 7, 2008, pp. 1032-43.

[3] A. B. Schwartz, X. T. Cui, D. J. Weber, D. W. Moran, "Brain Controlled Interfaces: Movement Restoration using Neural Prosthetics", Neuron, vol. 52, October 2006, pp. 205-220.

[4] M. A. Lebedev, Nicoleis, "Brain-machine interface: Past, present and future", Trends Neurosci. vol. 29, no.9, September 2006, pp. 536-546.

[5] W. Besio, K. Koka, R. Patwardhan, "Computer simulation and tank experimental verification of concentric ring electrodes", Engineering in Medicine and Biology Society, IEMBS'04. 26th Annual International Conference of the IEEE, vol. 1, September 2004, pp. 2243-2246.

[6] A. Rakotomamonjy, V. Guigue, G. Mallet, V. Alvarado, "Ensemble of svms for improving brain computer interface p300 speller performances", Int. Conf. on Artificial Neural Networks, 2005.

[7] R. C. Caracillo, M. C. F. Castro, "Classification of executed upper limb movements by means of EEG", Biosignals and Biorobotics Conference (BRC), ISSNIP, February 2013, pp. 1-6.

[8] B. Xu, A. Song, J. Wu, "Algorithm of imagined left-right hand movement classification based on wavelet transform and AR parameter model", Bioinformatics and Biomedical Engineering. ICBBE. The 1st International Conference on, July 2007, pp. 539-542.

[9] A. K. Mohamed, T. Marwala, L. R. John, "Single-trial EEG discrimination between wrist and finger movement imagery and execution in a sensorimotor BCI", Annual International Conference of the IEEE Engineering in Medicine and Biology Society, EMBS, 2011, pp. 6289-6293.

[10] Y. U. Khan, "Imagined wrist movement classification in single trial EEG for brain computer interface using wavelet packet". International Journal of Biomedical Engineering and Technology, vol. 4, no. 2, 2010, pp. $169-180$.

[11] T. Matsunanga, Y. Katayama, T. Hayami, K. Iramina, "Measurement of $\mathrm{mu} /$ beta ERD and gamma ERS during the imagination of body parts movement", $30^{\text {th }}$ Annual International IEEE EMBS Conference Vancouver Canada, August 2008.

[12] X. Yu, P. Chum, K. B. Sim, "Analysis the effect of PCA for feature reduction in non-stationary EEG based motor imagery of BCI system", Optik-International Journal for Light and Electron Optics, vol. 125, no. 3, 2014, pp. 1498-1502.

[13] X. Guo, X. Wu, X. Gong, L. Zhang, "Envelope detection based on online ICA algorithm and its application to motor imagery classification", Neural Engineering (NER), 6th International IEEE/EMBS Conference on, November 2013, pp. 1058-1061.

[14] M. Pal, S. Bhattacharyya, A. Konar, D. N. Tibarewala, R. Janarthanan, "Decoding of Wrist and Finger Movement from Electroencephalography Signal", IEEE International Conference on Electronics, Computing and Communication Technologies, Bangalore, January 2014. (in press)

[15] Y. Wang, V. Gonuguntla, G. Shafiq, K. C. Veluvolu, "BMFLC with neural network and DE for better event classification", Brain-Computer Interface (BCI), 2013 International Winter Workshop on, February 2013, pp. 34-35.

[16] S. Bhattacharyya, P. Rakshit, A. Konar, D. N. Tibarewala, R. Janarthanan, "Feature Selection of Motor Imagery EEG Signals Using Firefly Temporal Difference Q-Learning and Support Vector Machine", Swarm, Evolutionary, and Memetic Computing, 2013, pp. 534-545.

[17] W. Y. Hsu, "Application of Quantum-behaved Particle Swarm Optimization to Motor Imagery EEG Classification", International Journal of Neural Systems, vol. 23, no. 6, 2013.

[18] P. Rakshit, S. Bhattacharyya, A. Konar, A. Khasnobish, D. N. Tibarewala, R. Janarthanan, "Artificial Bee Colony Based Feature Selection for Motor Imagery EEG Data", Proceedings of Seventh Int. Conf. on Bio-Inspired Computing: Theories and Applications, January 2013, pp. 127-138.

[19] G. Dornhege, "Towards Brain-Computer Interfacing", 2007, MIT Press.

[20] K. Shenoi, B. Agrawal, "A design algorithm for constrained equiripple digital filters", Acoustics, Speech and Signal Processing, IEEE Trans. on, vol. 30, no.2, 1982, pp. 206-211.

[21] N. H. Phuc, T. Q. Khanh, N. N. Bon, "Discrete wavelets transform technique application in identification of power quality disturbance", Proceedings of the 9th Conference on Science and Technology, Ho Chi Minh, Vietnam, October 2005.

[22] K. M. Passino, "Biomimicry of bacterial foraging for distributed optimization and control", Control Systems, IEEE, vol. 22, no.3, 2002, pp. 52-67.

[23] S. Bhattacharyya, A. Sengupta, T. Chakraborti, A. Konar, D. N. Tibarewala, "Automatic feature selection of motor imagery EEG signals using differential evolution and learning automata", Medical \& biological engineering \& computing, 2013, pp. 1-9.

[24] J. J. Remus, K. D. Morton, P. A. Torrione, S. L. Tantum, L. M. Collins, "Comparison of a distance-based likelihood ratio test and k-nearest neighbor classification methods", IEEE Workshop on Machine Learning for Signal Processing, 16-19 Oct. 2008, pp.362-367. 